\title{
Treatment with Candida albicans biotherapic influences in vitro fungal adhesion to Ma-104 cells
}

\author{
Beatriz Guerreiro Basílio Costa, Camila Monteiro Siqueira, Gleyce Moreno \\ Barbosa, Venicio Feo da Veiga, Maristela Barbosa Portela, Rosangela Maria \\ de Araújo Soares, Carla Holandino
}

Federal University of Rio de Janeiro, Rio de Janeiro - RJ - Brazil

\begin{abstract}
Background: Oral candidiasis is an opportunist fungal infection in humans, mainly caused by Candida albicans. It occurs when the host presents an imbalance in the immune system and Candida spp., normally found in human flora, become able to develop the infection [1]. This disease is very common in HIV patients, and in all individuals that present immunossupression, such as patients treated with chemotherapy. Considering this scenario, the development of new medicines to treat oral candidiasis is mandatory.
\end{abstract}

Aims: The aim of this study was to evaluate citotoxicity, morphology and quantify the adhesion rates of $C$. albicans to biotherapic-treated Ma104 cells.

Methodology: The biotherapic was prepared following the Roberto Costa technique and Brazilian Homeopathic Pharmacopeia protocol [2]. Briefly, biotherapic 1X was prepared with $1 \mathrm{~mL}$ of aqueous solution containing $10^{8}$ yeasts of living Candida albicans plus $9 \mathrm{ml}$ of sterile distilled water. This solution was submmited to 100 mechanical succussions. Biotherapic $2 \mathrm{X}$ was obtained after addition of $1 \mathrm{ml}$ of $1 \mathrm{X}$ solution in $9 \mathrm{ml}$ of sterile distilled water and it was also submitted to 100 mechanical succussions. This procedure was repeated until biotherapic 30X was obtained. As a control, sterile dynamized water (30X) was used. The inhibition of fungal growth induced by biotherapic was evaluated by MTT method after 24 hours of treatment. The morphological aspects of Ma104-biotherapic-treated cells were analyzed by Giemsa staining after 5, 10 and 60 days, and compared with control groups (water 30X and untreated cells). Additionally, Ma104 cells were treated during 5 and 30 days with biotherapic in parallel with respective controls, and the index adhesion of yeast cells was quantified.

Results: The biotherapic was not able to reduce the viability of treated $C$. albicans when compared with controls. On the other hand, Ma104 treated cells presented important morphological alterations after 60 days, such as: cytoplasmic vacuoles, halos around the nucleolus and elongation of the plasmatic membrane. These changes were not observed in ,untreated cells nor in ones treated with water 30X. The adhesion index to Ma104 cells was reduced around 27\% after 5 and 30 days of treatment when compared to controls.

Conclusion: These results showed that the biotherapic did not present any citotoxicity, but was able to modify the morphological aspects of Ma-104 cells. Additionally, the interaction between host cells and ethilogic agent is directly influenced by biotherapic treatment, suggesting a promising antifungal potential of this medicine.

Key words: Candida albicans, biotherapic, toxicity, adhesion index. 


\section{References:}

[1] Farah CS et al. Oral candidiasis: Clinics in Dermatology, 18:553-562, 2000.

[2] Brazilian Homeopathic Pharmacopoeia, 2nd ed. SP: Atheneu, 1997.

\section{Tratamento com bioterápico Candida albicans influencia a adesão de fungos a células Ma104 in vitro}

\section{RESUMO}

Introdução: A candidíase oral é uma infecção fúngica oportunista em humanos, causada principalmente por Candida albicans. Esta doença ocorre quando o hospedeiro apresenta um desequilíbrio no sistema imune e Candida spp., normalmente encontrada na flora humana, torna-se capaz de desenvolver a infecção [1]. Esta patologia é muito comum em pacientes HIV e em todos indivíduos que apresentam imunossupressão, como pacientes submetidos à quimioterapia. Considerando este cenário, o desenvolvimento de novos medicamentos para tratamento de candidíase oral é extremamente importante.

Objetivos: O objetivo do estudo foi avaliar a citotoxidade, morfologia e quantificar o índice de adesão de $C$. albicans a células Ma104 tratadas com bioterápico.

Metodologia: O bioterápico foi preparado de acordo com a técnica Roberto Costa e o protocolo descrito na Farmacopéia Homeopática Brasileira [2]. $1 \mathrm{~mL}$ de solução aquosa contendo $10^{8}$ leveduras viáveis de Candida albicans mais $9 \mathrm{~mL}$ de água destilada estéril foram submetidos a 100 sucussões mecânicas para obtenção do bioterápico $1 \mathrm{X}$. O bioterápico $2 \mathrm{X}$ foi obtido após adição de $1 \mathrm{~mL}$ da solução $1 \mathrm{X}$ em $9 \mathrm{~mL}$ de água destilada estéril e também submetido a 100 sucussões mecânicas. Este procedimento foi repetido até obtenção do bioterápico 30X. Como controle, foi utilizada água dinamizada estéril (30X). A inibição do crescimento fúngico induzida pelo bioterápico foi avaliada por MTT após 24 horas de tratamento. Os aspectos morfológicos de células Ma104 tratados com bioterápico foram avaliados por coloração de Giemsa após 5, 10 e 60 dias, e comparados com os controles (água 30X e células não tratadas). Adicionalmente, células Ma104 foram tratadas durante 5 e 30 dias com bioterápico em paralelo com os respectivos controles, e o índice de adesão das leveduras foi quantificado.

Resultados: O bioterápico não foi capaz de reduzir a viabilidade de C. albicans tratada quando comparada aos controles. Em contrapartida, células Ma104 tratadas apresentaram importantes alterações morfológicas após 60 dias como: vacúolos citoplasmáticos, halos ao redor do nucléolo e prolongamentos da membrana plasmática. Estas mudanças não foram observadas em células não tratadas nem naquelas tratadas com água 30X. O índice de adesão a células Ma104 foi reduzido em aproximadamente $27 \%$ após 5 e 30 dias de tratamento quando comparado aos controles.

Conclusão: Estes resultados mostraram que o bioterápico não apresentou citotoxidade, mas foi capaz de modificar aspectos morfológicos de células Ma104. Adicionalmente, a interação entre células hospedeiras e o agente etiológico é diretamente influenciada pelo tratamento com bioterápico, sugerindo um potencial antifúngico promissor deste medicamento. 
Palavras-chave: Candida albicans, bioterápico, toxidade, índice de adesão

\section{(c) EY-NC-ND Licensed to GIRI}

Support: this study was partially funded by FAPERJ.

Conflict of interest: authors declare there is no conflict of interest

Correspondence author: Gleyce Moreno Barbosa, gleyce.moreno@gmail.com, http://sites.google.com/site/labmcf

How to cite this article Costa BGB, Siqueira CM, Barbosa GM, Veiga VF, Portela MB, Soares RMA, Hoalndino C. Treatment with Candida albicans biotherapic influences in vitro fungal adhesion to Ma-104 cells. Int J High Dilution Res [online]. 2011 [cited YYYY Month dd]; 10(36): 152-154. Proceedings of the XXV GIRI Symposium and VIII CBFH; 2011 Sep 04-07; Foz do Iguaçu (Brazil). GIRI and ABFH; 2011; Available from: http://www.feg.unesp.br/ ojs/index.php/ijhdr/article/view/487/499 